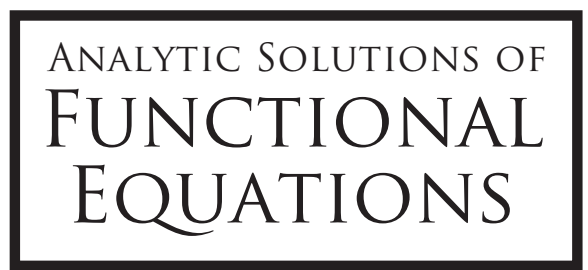


This page intentionally left blank 


\section{ANALYTIC SOLUTIONS OF FUNCTIONAL EQUATIONS}

\section{SUI SUN CHENG}

NationalTsing Hua University, R. O. China

WENRONG LI

Binzhou University, P. R. China 


\section{Published by}

World Scientific Publishing Co. Pte. Ltd.

5 Toh Tuck Link, Singapore 596224

USA office: 27 Warren Street, Suite 401-402, Hackensack, NJ 07601

UK office: 57 Shelton Street, Covent Garden, London WC2H 9HE

\section{British Library Cataloguing-in-Publication Data}

A catalogue record for this book is available from the British Library.

\section{ANALYTIC SOLUTIONS OF FUNCTIONAL EQUATIONS}

Copyright $(92008$ by World Scientific Publishing Co. Pte. Ltd.

All rights reserved. This book, or parts thereof, may not be reproduced in any form or by any means, electronic or mechanical, including photocopying, recording or any information storage and retrieval system now known or to be invented, without written permission from the Publisher.

For photocopying of material in this volume, please pay a copying fee through the Copyright Clearance Center, Inc., 222 Rosewood Drive, Danvers, MA 01923, USA. In this case permission to photocopy is not required from the publisher.

ISBN-13 978-981-279-334-8

ISBN-10 981-279-334-8 


\section{Preface}

Functions are used to describe natural processes and forms. By means of finite or infinite operations, we may build many types of 'derived' functions such as the sum of two functions, the composition of two functions, the derivative function of a given function, the power series functions, etc.

Yet a large number of natural processes and forms are not explicitly given by nature. Instead, they are 'implicitly defined' by the laws of nature. Therefore we have functional equations (or more generally relations) involving our unknown functions and their derived functions.

When we are given one such functional equation as a mathematical model, it is important to try to find some or all solutions, since they may be used for prediction, estimation and control, or for suggestion of alternate formulation of the original physical model. In this book, we are interested in finding solutions that are 'polynomials of infinite order', or more precisely, power series functions.

There are many reasons for trying to find such solutions. First of all, it is sometimes 'obvious' from experimental observations that we are facing with natural processes and forms that can be described by 'smooth' functions such as power series functions. Second, power series functions are basically 'generated by' sequences of numbers, therefore, they can easily be manipulated, either directly, or indirectly through manipulations of sequences. Indeed, finding power series solutions are not more complicated than solving recurrence relations or difference equations. Solving the latter equations may also be difficult, but in most cases, we can 'calculate' them by means of modern digital devices equipped with numerical or symbolic packages! Third, once formal power series solutions are found, we are left with the convergence or stability problem. This is a more complicated problem which is not completely solved. Fortunately, there are now several standard techniques which have been proven useful.

In this book, basic tools that can be used to handle power series functions and analytic functions will be given. They are then applied to functional equations in which derived functions such as the derivatives, iterates and compositions of the unknown functions are involved. Although there are numerous functional equations in the literature, our main objective is to show by introductory examples how analytic 
solutions can be derived in relatively easy manners.

To accomplish our objective, we keep in mind that this book should be suitable for the senior and first graduate students as well as anyone who is interested in a quick introduction to the frontier of related research. Only basic second year advanced engineering mathematics such as the theory of a complex variable and the theory of ordinary differential equations are required, and a large body of seemingly unrelated knowledge in the literature is presented in an integrated and unified manner.

A synopsis of the contents of the various chapters follows.

- The book begins with an elementary example in Calculus for motivation. Basic definitions, symbols and results are then introduced which will be used throughout the book.

- In Chapter 2, various types of sequences are introduced. Common operations among sequences are then presented. In particular, scalar, term by term, convolution and composition products and their properties are discussed in detail. Algebraic derivation is also introduced.

- Power series functions are treated as generating functions of sequences and their relations are fully discussed. Stability properties are discussed and Cauchy's majorant method is introduced. The Siegel's lemma is an important tool in deriving majornats.

- In Chapter 4, the basic implicit function theorem for analytic functions is proved by Newton's binomial expansion theorem. Schröder and Poincaré type implicit functions together with several others are discussed. Application of the implicit theorems for finding power series solutions of polynomial or rational type functional equations are illustrated.

- In Chapter 5 analytic solutions for several classic ordinary differential equations or systems are derived. The Cauchy-Kowalewski existence theorem for partial differential equations is treated as an application. Then several selected functional differential equations are discussed and their analytic solutions found.

- In Chapter 6 analytic solutions for functional equations involving iterates of the unknown functions (or more general composition with other known functions) are treated. These equations are distinguished by whether derivatives of the unknown functions are involved. The last section is concerned with the existence of power solutions.

Some of the material in this book is based on classical theory of analytic functions, and some on theory of functional equations. However, a large number of material is based on recent research works that have been carried out by us and a number of friends and graduate students during the last ten years.

Our thanks go to J. G. Si, X. P. Wang, T. T. Lu and J. J. Lin for their hard works and comments. We would also like to remark that without the indirect help 
of many other people, this book would never have appeared.

We tried our best to eliminate any errors. If there are any that have escaped our attention, your comments will be much appreciated. We have also tried our best to rewrite all the material that we draw from various sources and cite them in our notes sections. We beg your pardon if there are still similarities left unattended or if there are any original sources which we have missed.

Sui Sun Cheng and Wenrong Li 
This page intentionally left blank 


\section{Contents}

Preface $\quad$ v

1. Prologue 1

1.1 An Example . . . . . . . . . . . . . . . . . . 1

1.2 Basic Definitions .................... 2

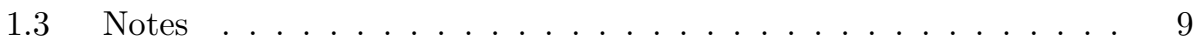

2. Sequences 11

2.1 Lebesgue Summable Sequences . . . . . . . . . . . . . . . . . . . 11

2.2 Relatively Summable Sequences . . . . . . . . . . . . . . . 18

2.3 Uniformly Summable Sequences . . . . . . . . . . . . . . . . . 21

2.4 Properties of Univariate Sequences . . . . . . . . . . . . . . 25

2.4.1 Common Sequences . . . . . . . . . . . . . . 25

2.4.2 Convolution Products . . . . . . . . . . . . . 26

2.4.3 Algebraic Derivatives and Integrals . . . . . . . . . . . 32

2.4.4 Composition Products .............. . . 34

2.5 Properties of Bivariate Sequences . . . . . . . . . . . . . . . . . . . 42

2.6 Notes ....................... . . 47

3. Power Series Functions 49

3.1 Univariate Power Series Functions . . . . . . . . . . . . . . . 49

3.2 Univariate Analytic Functions . . . . . . . . . . . . . . . . 56

3.3 Bivariate Power Series Functions . . . . . . . . . . . . . . 63

3.4 Bivariate Analytic Functions . . . . . . . . . . . . . 67

3.5 Multivariate Power Series and Analytic Functions . . . . . . . . . . 68

3.6 Matrix Power Series and Analytic Functions . . . . . . . . . . . . . 71

3.7 Majorants ... . . . . . . . . . . . . . . . 72

3.8 Siegel's Lemma . . . . . . . . . . . . . . . . . . 77

3.9 Notes ...................... 82 
4. Functional Equations without Differentiation 83

4.1 Introduction . . . . . . . . . . . . . . . . . . 83

4.2 Analytic Implicit Function Theorem . . . . . . . . . . . . . . . 86

4.3 Polynomial and Rational Functional Equations . . . . . . . . . . . 90

4.4 Linear Equations . . . . . . . . . . . . . . . . . . . . 100

4.4 Equation I . . . . . . . . . . . . . . . . . . 100

4.4 .2 Equation II . . . . . . . . . . . . . . . . . . . . 102

4.4.3 Equation III . . . . . . . . . . . . . . . . . . . 103

4.4.4 Equation IV . . . . . . . . . . . . . . . . . . 105

4.4.5 Equation V. . . . . . . . . . . . . . . . 107

4.4.6 Schröder and Poincaré Equations . . . . . . . . . . . . 110

4.5 Nonlinear Equations . . . . . . . . . . . . . . . . . . 114

4.6 Notes ......................... 121

5. Functional Equations with Differentiation 123

5.1 Introduction . . . . . . . . . . . . . . . . . . 123

5.2 Linear Systems . . . . . . . . . . . . . . . . . . . . . . . . . . . . . . . . . . . . . . . . . .

5.3 Neutral Systems . . . . . . . . . . . . . . . . . . 128

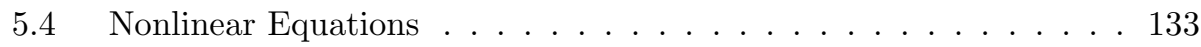

5.5 Cauchy-Kowalewski Existence Theorem . . . . . . . . . . . . . 139

5.6 Functional Equations with First Order Derivatives . . . . . . . . . 141

5.6 .1 Equation I . . . . . . . . . . . . . . . . . . . . . . . 142

5.6 .2 Equation II . . . . . . . . . . . . . . . . . . . . . . . . . . . . . . . . . . . . . . . . . . . . . . . .

5.6 .3 Equation III . . . . . . . . . . . . . . . . . . . . . . 145

5.6 .4 Equation IV . . . . . . . . . . . . . . . . . . . . 147

5.6 .5 Equation V..................... . . 148

5.6 .6 Equation VI . . . . . . . . . . . . . . . . . . 150

5.7 Functional Equations with Higher Order Derivatives . . . . . . . . 152

5.7.1 Equation I . . . . . . . . . . . . . . . . . . . . . 153

5.7 .2 Equation II . . . . . . . . . . . . . . . . . . . . 154

5.7 .3 Equation III . . . . . . . . . . . . . . . . . . 156

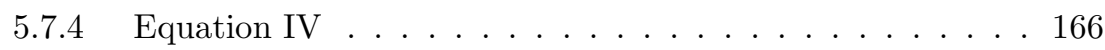

5.8 Notes . . . . . . . . . . . . . . . . . . 170

6. Functional Equations with Iteration $\quad 175$

6.1 Equations without Derivatives . . . . . . . . . . . 175

6.1.1 Babbage Type Equations . . . . . . . . . . . . . 176

6.1.2 Equations Involving Several Iterates . . . . . . . . . . . . . . . 182

6.1.3 Equations of Invariant Curves . . . . . . . . . . . . 190

6.2 Equations with First Order Derivatives . . . . . . . . . . . 197

6.2.1 Equation I . . . . . . . . . . . . . . . . . . . . . . . 198

6.2 .2 Equation II . . . . . . . . . . . . . . . . . . 202 
6.2 .3 Equation III . . . . . . . . . . . . . . . . . . . . 206

6.2.4 Equation IV . . . . . . . . . . . . . . . . . . . . . . . . . . 212

6.2.5 First Order Neutral Equation . . . . . . . . . . . . 214

6.3 Equations with Second Order Derivatives . . . . . . . . . . . 222

6.3.1 Equation I . . . . . . . . . . . . . . . . . . . . 223

6.3.2 Equation II . . . . . . . . . . . . . . . . . . . . 230

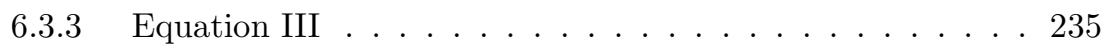

6.3.4 Equation IV . . . . . . . . . . . . . . . . . 240

6.4 Equations with Higher Order Derivatives . . . . . . . . . . . . . 244

6.4.1 Equation I . . . . . . . . . . . . . . . . . . . . . 247

6.4.2 Equation II . . . . . . . . . . . . . . . . . . . 249

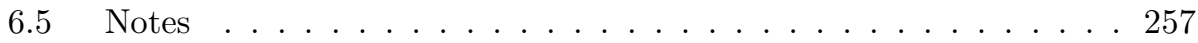

Appendix A Univariate Sequences and Properties 259

A.1 Common Sequences . . . . . . . . . . . . . . . 259

A.2 Sums and Products . . . . . . . . . . . . . . . 260

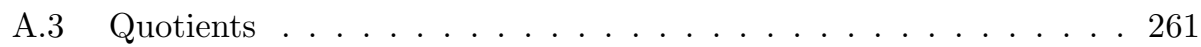

A.4 Algebraic Derivatives and Integrals . . . . . . . . . . . . . . 261

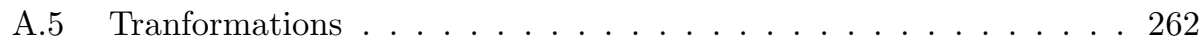

A.6 Limiting Operations . . . . . . . . . . . . . . . . 263

A.7 Operational Rules . . . . . . . . . . . . . . . 263

A.8 Knowledge Base . . . . . . . . . . . . . . . 266

A.9 Analytic Functions . . . . . . . . . . . . . . . . 267

A.10 Operations for Analytic Functions . . . . . . . . . . 267

$\begin{array}{ll}\text { Bibliography } & 271\end{array}$

$\begin{array}{ll}\text { Index } & 283\end{array}$ 